neglected, even though it appear already perfectly re-adapted; otherwise, small portions of surface matter might lodge beneath, and, by distending the wound-channel, give rise to irritation.

After the operation has been fully terminated, I think it further advisable to wait half a minute or so, and thereupon by pressure to empty the chamber of the small quantity of fluid freshly secreted. This fluid has not only sometimes small fragments of surface matter suspended in it, but mostly also a slight admixture of blood.

[Every operator knows that the trial of the vision which it is quite proper to make for the reassurance of the patient after the operation is finished, does not succeed in all cases. This, unless a continuous mass of surface matter was left behind, arises either from the patient being momentarily dazzled, or from a yellowish discoloration of the aqueous humour by blood. In the latter case, when, by gentle pressure, the drop of aqueous humour has been made to escape, the trial of the vision proves perfectly successful. I do not imagine that this turbid aqueous humour is possessed of any deleterious qualities. But I think that, even where the vision is satisfactory, some advantage attaches to the evacuation of the humour secreted under the direct influence of the traumatic irritation.]

[To be continued.]

\section{ON A REMARKABLE ALTERATION OF APPEARANCE AND STRUCTURE OF THE HUMAN HAIR.}

\section{By ERASMUS WILSON, F.R.S.*}

I HAVE the honour of submitting to the Royal Society a specimen of human hair of very remarkable appearance. Every hair is brown and white in alternate bands, looking as if encircled with rings; and this change of aspect extends throughont the whole length of the hair, and gives to the general mass a curiously speckled character. The brown segment of the hair, which represents its normal colour, measures about one-fiftieth of an inch in length, or something less than a quarter of a line; the white, or abnormal segment, about half that length-namely, one-hundredth of an inch; and the two together about one thirty-sixth of an inch, cr one-third of a line.

The hair was taken from a lad aged seven years and a half, a gentleman's son. He is reported as being "an active healthy boy, quick and intelligent." $\mathrm{He}$ was delicate up to the age of four, having suffered in quick succession the diseases of childhood, a severe attack of croup, and several attacks of convulsions. The change in the appearance of the hair was first noticed when he was between two and three years old; and has increased perceptibly during the last two years. There is no similar alteration of structure of the eyebrows and eyelashes; his complexion is dark, while that of a younger brother is fair; and the latter is free from any alteration of the hair.

Examination of the hair with a lens shows that the cylinder of the hair is perfectly uniform; that the white portion is contained within the cuticle, and occupies the whole breadth of the cylinder; whilst it frequently presents a rounded cone at the central ex-

- Read at the Royal Booiety, Mareh 1867. tremity, and breaks up into fibres at the opposite or distal end; and in some instances this fibrous struc. ture is apparent at both ends of the white segment. Moreover, by transmitted light, the white segment is found to be opaque, and consequently presents a dark shade, while the intermodiate or brown portion has the transparency of normal hair.

When the transparency of the hair is increased by immersion in Canada balsam slightly diluted with spirits of turpentine, the white and opaque segment is reduced in dimensions, and is rendered more or less transparent by imbibition of the volatile fluid. Moreover, it is clearly demonstrated by this process, that the opacity of the segment, its whiteness when seen by reflected light, and its darkness by transmitted light, are all due to the presence of spaces in the fibrous portion of the hair, filled with airglobules. The air-spaces are nocosenily very numerous and assembled closely together; while at the ends of the white segment they have more or less of a linear arrangement, and give a fibrons appearance to the opaque mass. Moreover, the partial transparency of the hair caused by the balsam demonstrates that, besides the air-spaces, large and small, contained in the opaque portion, minute air-spaces, sometimes arranged in linear order, and sometimes communicating and forming short, irregular canals, are also met with in the transparent part of the hair. And, in addition to the minute air-spaces of the plates of the fibrous portion of the hair, an accumulation of air-globules is also very apparent in the cells of the medulla.

It is evident from this examination of the hairs, that they are imperfect in structure and development, and that their imperfection indicates a weak producing organ, and probably a weakly constitution of the individual; that the cells of which the fibrous portion of the hair is composed, instead of being filled with a horny plasma, are tinged with aqueous fluid, and the desiccation of this fluid leaves behind it vacuities which, in the subsequent growth of the shaft, become filled with air. The most renarkable plenomenon in connexion with the case, however, is the alternation of imperfect and perfect cells; the period of continuance of the two processes, supposing them to be equally active in point of time, being twice as long for the perfect as for the imperfect structure.

Since the publication of the observations of Berthold in Müller's Archiv for 1850, it is generally believed that the hair grows faster during the day than during the night. Hence the first suggestion that occurred to me in connexion with the present case, seeing that the white or opaque segment was shorter by one-half than the brown, was that the former represented the slower growth by night, and the latter the quicker growth by day; the white and the brown together representing an entire day of twenty-four hours. But other observations by myself have given, as the average growth of the hair of the head in persons who had been shaved, one-elghth of an inch for the week, and consequently one fiftysixth of an inch for the twenty-four hours. Now, the length of hair comprehended by the white and the brown in the present case is one thirty-sixth of an inch, and consequently a much more active growth than is normally met with-corresponding, in fact, in a similar ratio, with thirty-seven hours instead of twenty-four.

I therefore refrain from speculating upon the cause of alternation of the healthy and morbid structure presented by this case, and restrict myself to the narration of the fact that, during a certain space of time, amounting to a day or more, the hair is produced of normal structure; while during another 
space of time, of undetermined extent, the hair is produced unhealthily; that the periods of bealthy formation correspond pretty accurately in extent, as do those of unhealthy formation; while the latter, in measurement, are only half as extensive as the former ; moreover, that the differences of the pathological operation are, the production of a horny plasma in the normal process, and of serous and watery cell-contents in the abnormal process.

I may further observe, that it is by no means improbable that the "dead" and faded hair which is met with after some illnesses, and in instances of debilitated health, may be due to a similar pathological process, although wanting in the periodicity and alternation which render the present case so remarkable.

\section{A CASE OF MULTILOCULAR OVARIAN TUMOUR SUCCESSFULLY REMOVED.}

By F. PAGE, M.D., F.R.C.S.E., Medical Oficec if the Portsea Island Union Hospital.

Elizabeth Garretr, aged 28, mother of four children, a thin spare woman, much emaciated and worn down, felt pain and swelling in the left side of the abdomen about three years since, after her last confinement. She was then admitted into the Ports. mouth Hospital for three or four months, and was tapped. She continued an out-patient to November last, having been tapped several times by officers of that establishment.

She was admitted into this hospital on November 14th. The abdomen was then of immense size; a large tumour of a pyramidal shape, very hard, occupied the abdomen in front. An attempt had been lately made to tap, but the contents, being of a semisolid character, would not escape; the confines of the tumour could be easily felt on each side, and, a large trocar having been introduced a little wide of the tumour on the left side, about three gallons of thick gruelly fluid ran off. This brought the tumour more distinctly into view, but did not lessen its size. It then seemed that this large hard tumour was distinct from the cyst containing the fluid, or that it was solid in front, and had some connection with it behind. The latter was the case.

From the hopeless condition of the patient, and the little chance there was of her long surviving, unless something was at once done, I suggested removing the tumour.

November 24th, 2 P.M. With the kind advice and assistance of my friends, Drs. Raper and Simpson, and Messrs. Pritchard and Gould, the patient having been chloroformed, an incision five or six inches long was made in the linea alba. This brought the tumour into view; but the adhesions were so firm in front that they could not be separated. A free opening was made into the tumour, and its contents, a thick gelatinous substance, with a number of cysts of all sizes, were baled out. A prolongation of the tumour up to the diaphragm, with strong adhesions underneath, was removed, and the lower solidified portion turned out with it; the pedicle, which sprang from the left side, was secured with strong silk and divided; some loose cellular tissue was removed; and when the oozing of blood had ceased, the edges of the wound, with the peritoneum also, were brought together by deep steel pins and super- ficial silver wire; a bandage was applied, and the patient placed in bed. When she had recovered from the effects of chloroform, three grains of opium were taken with brandy, and a grain of opium and a teaspoonful or two of brandy in iced water were ordered to be given every three hours.

November 24th, 10 P.M. Pulse 112 ; she was comfortable, free from pain; the countenance was good; she had passed about twelve ounces of urine; the abdomen was not distended; she complained of thirst.

The progress of the case was steadily favourable throughout. On the 28th, the wound was found to have united by the first intention, and the superficial sutures were removed on the 29 th. Opium was given in grain doses at intervals varying from four hours to twice daily, and ultimately in two grain doses every night.

On December 20th, the patient was convalescent; there had been slight suppuration, but the opening was now closed; she was rapidly recovering her health and strength.

She was discharged cured on December 31st.

REMARKs This case is interesting in one or two points. When the tumour was first examined, and when brought more distinctly into view by the tapping, it seemed perfectly circumscribed; this arose from the strong adhesions in front, and the dense tissue forming this part of the tumour. Above the apparent confines, the prolongation was not at all attached in front; consequently the tumour proved to be actually double the size it was at first supposed. The adhesions in front being so extensive and so firm, I thought it better, first to empty it, and, as it was loose behind, when the upper part was removed those in front readily gave way, and the whole turned out without much trouble, The mass was very large, and consisted of numerous cysts of all sizes, with substances of various consistence. There was an opening from the back of the hard cyst into the large one behind, whence escaped about a gallon of fluid; this was the cyst from which three gallons of fluid had been drawn a week before the extirpation.

Hohse-Pox. M. Chauveau read a memoir at the February meeting of the Lyons Imperial Society of Medicine on Vaccine Infection by the Respiratory Organs. He referred to a previous memoir, in which he demonstrated that the development of the generalised vaccine exanthema does not depend on an asserted spontaneity of the disease, but is connected with the absorption of the virus by another channel than the skin. He has been able to produce the generalised exanthema by injecting the virus directly into the circulatory system; which fact has led him to admit that, in the so-called spontaneous cases amongst animals, the vaccination was due to absorption, through the respiratory organs, of the virus, when in a pulverulent state and in suspension in the air inspired. In order to verify this hypothesis, $M$. Chauveau tried on horses three experiments, of introducing the vaccine matter by the respiratory organs. Two had no apparent result. In the third case, by the aid of the capillary cannula, a drop of vaccine matter was dropped into the trachea; and a week afterwards some dried vaccine powder was also inserted into the trachea. The animal died eighteen days afterwards ; and, at the autopsy, four well developed pustules were found around the lips. The trachea was perfectly free from eruption. M. Chauveau, therefore, attributes the so-called spontaneous development of horse-pox to the introduction of virus by the respiratory organs. - Gazette Médicale de Lyon. 